hep-th/0609138

\title{
Renormalization Ambiguities and Conformal Anomaly in Metric-Scalar Backgrounds
}

\author{
M. Asorey \\ Departamento de Física Teorica, Universidad de Zaragoza, 50009, Zaragoza, Spain \\ G. de Berredo-Peixotd $\circledast$ and I. L. Shapird丹 \\ Departamento de Física - ICE, Universidade Federal de Juiz de Fora, Juiz de Fora, 36036-330, MG, Brazil
}

\begin{abstract}
We analyze the problem of the existing ambiguities in the conformal anomaly in theories with external scalar field in curved backgrounds. In particular, we consider the anomaly of self-interacting massive scalar field theory and of Yukawa model in the massless conformal limit. In all cases the ambiguities are related to finite renormalizations of a local non-minimal terms in the effective action. We point out the generic nature of this phenomenon and provide a general method to identify the theories where such an ambiguity can arise.
\end{abstract}

PACS numbers: $04.62 .+\mathrm{v}, 11.10 . \mathrm{Hi}, 11.10 . \mathrm{Jj}$

Keywords: Curved space-time, Non-Minimal Interaction, Renormalization Group, Conformal Anomaly

\section{INTRODUCTION}

Fundamental scalar fields constitute an important element of the standard model of particle physics and modern cosmology, in spite that there is not yet any experimental confirmation of their existence. Indeed, the cosmological applications of the scalar fields (e.g. inflaton [1], cosmon [2] and quintessence [3]) require formulating them on curved backgrounds at both classical and quantum level. It is well known that the consistent description of scalar field on curved backgrounds (see, e.g. [4] for an introduction) is based on the non-minimal covariant action $S=S_{s c a l}+S_{v a c}$, where

$$
\begin{aligned}
S_{\mathrm{scal}} & =\frac{1}{2} \int d^{4} x \sqrt{g}\left\{g^{\mu \nu} \partial_{\mu} \phi \partial_{\nu} \phi+m^{2} \phi^{2}+\xi R \phi^{2}\right. \\
& \left.-\frac{\lambda}{12} \phi^{4}+\tau_{1} \square \phi^{2}-\frac{\tau_{2}}{2} m \phi^{3}-\kappa m^{2} \phi-\rho m \phi R\right\},
\end{aligned}
$$

and

$$
\begin{aligned}
& S_{v a c}=\int d^{4} x \sqrt{g}\left\{-\frac{1}{16 \pi G}(R+2 \Lambda)+a_{1} C^{2}\right. \\
& \left.+a_{2} R^{2}+a_{3} E+a_{4} \square R\right\} \text {. }
\end{aligned}
$$

Here $C^{2}=R_{\mu \nu \alpha \beta}^{2}-2 R_{\alpha \beta}^{2}+1 / 3 R^{2}$ is the square of the Weyl tensor and $E=R_{\mu \nu \alpha \beta}^{2}-4 R_{\alpha \beta}^{2}+R^{2}$ is the integrand of the Gauss-Bonnet topological invariant (Euler number). $a_{1}, \ldots, a_{4}$ and $G, \Lambda$ are independent parameters of the vacuum action, $\lambda, \xi, \tau_{1}, \tau_{2}, \kappa, \rho$ are independent parameters in the scalar sector, including the non-minimal parameters $(\xi, \rho)$ of interaction with the scalar curvature [31]. The action $S$ contains all local diffeomorphism invariants up to dimension four to guarantee the renormalizability of the theory at quantum level. Terms with

\footnotetext{
*Electronic address: guilherme@fisica.ufjf.br

${ }^{\dagger}$ On leave from Tomsk State Pedagogical University, Russia; Electronic address: shapiro@fisica.ufjf.br
}

odd powers of $\phi$ have been included to consider models with spontaneous breaking of the $\phi \leftrightarrow-\phi$ symmetry [32].

The presence of non-minimal terms like $R \phi^{2}$ and $R \phi$ and $S_{v a c}$ might be required because they may emerge as infinite quantum corrections. In this case one needs such terms in the classical action for renormalization purposes. These are not, however, the only possible types of quantum loops contributions. Some other important quantum corrections to $S$ of non-local type might also appear. In the absence of scalar fields, for the massless conformal case, these contributions can be partially evaluated by means of the conformal anomaly (see, e.g. 5, 6, 7, 8] for some works on conformal anomaly and [9, 10] for the anomaly-induced effective action). In the case of massive quantum fields there is no general method of calculation and one has to rely on some expansions which are valid, typically, either for large or small masses. In any case the effective action is a functional of the metric and the background scalar field, and the quantum corrections are somehow related to the renormalization of the parameters $a_{i}, G, \Lambda$.

There are, however, more involved situations, where the scalar field is present at the cosmic scale. In this case the quantum fields are interacting not only to the external metric but also (directly or via the metric) to the external scalar field. Therefore, in order to provide renormalizability, the vacuum effective action has to include all local diffeomorphism invariants of dimension four constructed from both metric and scalar fields. In other words the whole scalar action $S_{\text {scal }}$ must be considered as a part of the vacuum action. In this situation it is important to extend the available information concerning conformal anomaly and corresponding derivation of the effective action to the theory with an external scalar. It is known that for vanishing external scalar fields the anomaly manifests an ambiguity in the $\square R$-sector 5,11 . In the recent paper [7] we have investigated this ambiguity in details. In particular, we have shown that there is no conflict between the results for the coefficient of the $\square R$ term in the dimensional and other regularizations, 
for the dimensional regularization leaves this coefficient completely arbitrary. Furthermore, we have constructed another example of covariant Pauli-Villars regularization with similar ambiguity. In all cases the coefficient of the $\square R$ term can be fixed by the renormalization condition for the finite $\int R^{2}$-term in the vacuum action. Thus the ambiguity concerns only the initial point of the renormalization group trajectory for the corresponding parameter but not the shape of this trajectory.

The purpose of the present paper is to extend the results of [7] for the case of a scalar field. We study the anomaly and the effective action in the presence of a non-vanishing scalar background and in particular investigate if new ambiguities appear in that case. Indeed, the scalar may also interact with other, e.g. fermion fields. Therefore, a complete understanding of the problem requires to consider also the quantum effects of these interactions. Finally, we consider two distinct models: selfinteracting scalar field theory and Yukawa model for the scalar-spinor interactions. In both cases we shall find the anomaly via the massless conformal limit in the effective action for massive fields, not only by using dimensional regularization [6] (see also [7]), but also by covariant Pauli-Villars regularization. The use of this regularization has proved very fruitful in the analysis of the anomalous ambiguity in the case of pure gravitational background [7] and we shall extend these results for the case with non-vanishing scalar field backgrounds.

The paper is organized as follows. In next section we formulate the conformal symmetry and anomaly for the scalar backgrounds in curved space-times. The derivation of anomaly is performed by means of dimensional regularization [6] and the discussion of corresponding ambiguities will parallel that of Ref. [7].

In sections 3 and 4 we analyze the divergence structure in the self-interacting scalar theory and in the Yukawa model. Furthermore, we use Barvinsky-Vilkovisky formalism [12, 13] and the approach developed in [14] to derive the non-local finite part of the effective action for the case of massive Yukawa model. In the case of self-interacting scalar theory, this calculation has been already done in [15]. The results for massive theories enable us to apply the covariant Pauli-Villars regularization for the comprehensive analysis of the nature of the new ambiguities due to the presence of scalar background. Finally, in section 5 we outline our conclusions concerning the general status of local conformal symmetry at quantum level.

\section{LOCAL CONFORMAL SYMMETRY AND DIMENSIONAL REGULARIZATION}

It is well known that the global conformal symmetry $S O(4,2)$ is usually broken by quantum radiative corrections. At the same time there is another similar symmetry associated to the background fields. This symmetry is local [33] and is not directly related to space-time trans- formations, however it is also broken by anomaly. The local conformal symmetry corresponds, in the case of the scalar field $\phi$, to the transformation law

$$
\phi \rightarrow \phi^{\prime}=\phi \cdot e^{-\sigma}, \quad g_{\mu \nu} \rightarrow g_{\mu \nu}^{\prime}=g_{\mu \nu} \cdot e^{2 \sigma}
$$

where $\sigma=\sigma(x)$ is an space-time scalar function. This local conformal symmetry reduces to Weyl symmetry when there is no scalar background fields $\phi$. The action $S$ is invariant under this symmetry if the following conditions are satisfied:

$$
m=0, \quad \xi=\frac{1}{6}, \quad \frac{1}{G}=0, \quad a_{2}=0 .
$$

The corresponding Noether identity is

$$
\mathcal{T}=-\frac{2}{\sqrt{g}} g_{\mu \nu} \frac{\delta S}{\delta g_{\mu \nu}}+\frac{1}{\sqrt{g}} \phi \frac{\delta S}{\delta \phi}=0 .
$$

The first term is the usual trace of the EnergyMomentum tensor while the second term is a new contribution due to the presence of a background scalar. If the scalar satisfies the classical equation of motion the second term vanishes and the Noether identity (5) reduces to the standard condition for Weyl invariance.

The existence of a quantum anomaly implies the violation of the identity (5). In absence of scalar background fields, the simplest way to discover the conformal anomaly is via dimensional regularization [ $[6]$. However, the derivation of the anomaly in dimensional regularization may exhibit some ambiguities [7] and these ambiguities might become even larger in the presence of scalar background fields.

Dimensional regularization proceeds by extending the covariant perturbative expansion to an arbitrary dimension $n$. This is achieved by replacing the differentials and integrals in the action (11) by the $n$-dimensional covariant derivatives and integrals, e.g.

$$
\begin{aligned}
\frac{1}{\sqrt{g}} \sum_{\mu, \nu=1}^{4} \partial_{\mu} g^{\mu \nu} \sqrt{g} \partial_{\nu} & \rightarrow \frac{1}{\sqrt{g}} \sum_{\mu, \nu=1}^{n} \partial_{\mu} g^{\mu \nu} \sqrt{g} \partial_{\nu} ; \\
\int d^{4} x & \rightarrow \int d^{n} x .
\end{aligned}
$$

The dimensionality of the different terms in the action for $n \neq 4$ is restored by inserting an appropriate power of the dimensionfull parameter $\mu$ which sets the renormalization scale.

The first observation is that the purely metric part of the action (2) is not conformal invariant in $n$ dimensions independent on the values of the parameters of the action, in both cases of global and local transformations. The situation in the scalar sector (1) is more complicated. In fact, the procedure (6) leaves the room for different prescriptions to extend the action of the scalar field. In order to satisfy the symmetry under the global conformal symmetry (17) one needs to comply with the same conditions (4). However, in order to provide the local conformal symmetry one has to generalize the transformation 
law for the scalar field and the value of the non-minimal parameter $\xi$, correspondingly, to

$$
\phi \rightarrow \phi^{\prime}=\phi \cdot \exp \left(\frac{2-n}{2} \sigma\right), \quad \xi(n)=\frac{n-2}{4(n-1)} .
$$

An important observation is that, in the classical theory, the introduction of conformal coupling is not really necessary. After the removal of the regularization by taking $n \rightarrow 4$ limit, the theory is the same independently on whether we follow (7) or leave the transformation for scalar in the original form (3) and keep $\xi=1 / 6$ in $n$ dimensions.

Now let us discuss the violation of conformal symmetry on quantum level in the framework of dimensional regularization. There are two alternative, although equivalent, ways of deriving the anomaly. Let us start from the one which is close to the original derivation of Duff [ $[\underline{6}]$ and is based on the direct use of the counterterms. The second method is based on the evaluation of the finite part of the effective action [17], which becomes anomalous after subtracting divergencies. This method will be used in next sections to check the correctness of the derivation based on the counterterms.

It proves useful to rewrite the Noether identity (5) in different field variables. Let us define $g_{\mu \nu}=\bar{g}_{\mu \nu} \cdot e^{2 \sigma}$ and $\phi=\bar{\phi} \cdot e^{-\sigma}$, where $\bar{g}_{\mu \nu}$ and $\bar{\phi}$ are some fiducial fields. It is important to keep the original four-dimensional form of the transformation law (31) for the scalar, instead of the generalized one (7). In the new variables the conformal transformation is reduced to the shift of $\sigma$, while (5) is cast in the new form

$$
\mathcal{T}=-\left.\frac{1}{\sqrt{\bar{g}}} \frac{\delta}{\delta \sigma} S\left(n ; \bar{g}_{\mu \nu} e^{2 \sigma}, \bar{\phi} e^{\frac{2-n}{2} \sigma}\right)\right|_{\substack{\sigma \rightarrow 0 \\ n \rightarrow 4}}=0,
$$

where the procedure includes also the replacements $\bar{g}_{\mu \nu} \rightarrow g_{\mu \nu}, \bar{\phi} \rightarrow \phi$.

At quantum level, the effective action which includes classical action plus the naive one-loop corrections $\Gamma=$ $S+\bar{\Gamma}^{(1)}$ is locally conformal invariant at $n$ space-time dimension. Now, the presence of the UV divergences in $\bar{\Gamma}^{(1)}$ requires introducing local counterterms, $\Delta S^{(1)}$, which explicitly break the conformal symmetry. In the vicinity of $n=4$ the requested counterterms are defined as

$$
\begin{aligned}
\Delta S^{(1)}\left(n, g_{\mu \nu}, \phi\right) & =\frac{1}{n-4} \int d^{4} x \sqrt{g}\left\{\beta_{1} C^{2}+\beta_{2} E+\beta_{3} \square R\right. \\
& \left.+\alpha_{1} R \phi^{2}+\alpha_{2} \square \phi^{2}+\alpha_{3} \phi^{4}\right\},
\end{aligned}
$$

$\beta_{i}$ and $\alpha_{i}$ being the $\beta$-functions in the vacuum and scalar matter sectors, respectively. The effect of these terms into conformal Noether identity is

$$
\begin{aligned}
& <\mathcal{T}>=-\left.\frac{1}{\sqrt{g}} \frac{\delta \Delta S^{(1)}\left(n ; g_{\mu \nu} e^{2 \sigma}, \phi e^{-\sigma}\right)}{\delta \sigma}\right|_{\substack{\sigma \rightarrow 0 \\
n \rightarrow 4}} \\
= & \beta_{1} C^{2}+\beta_{2} E+\beta_{3} \square R+\alpha_{1} R \phi^{2}+\alpha_{2} \square \phi^{2}+\alpha_{3} \phi^{4} .
\end{aligned}
$$

Let us remark that this expression is exactly the anomaly of the global conformal symmetry and hence it can be obtained via the renormalization group [4, 18] or the $\zeta$ regularization method [19]. The expression for the conformal anomaly (10) has been used to get an explicit form of the anomaly-induced action in the scalar-metric theory (cosmon model) [20], with very interesting phenomenological implications. In next sections we will show that the approach described above agrees with the calculation based on the heat-kernel [12] and Pauli-Villars methods.

Before discussing the source of ambiguities in the dimensional regularization, let us make some general remarks. The counterterms in $\Delta S$ are local expressions on the background fields, whereas among the finite terms of the effective action $\bar{\Gamma}^{(1)}$ there are both local and nonlocal terms. The universality of non-local terms [21, 22] guarantees the universality of the terms in the anomaly which are not total derivatives. In the particular case of metric and scalar background fields this means that the ambiguities should only affect the coefficients $\alpha_{2}$ and $\beta_{4}$ of the terms $\square \phi^{2}$ and $\square R$ and the corresponding local terms $R \phi^{2}$ and $R^{2}$ in the finite part of the effective action. The ambiguities arise from the conformally invariant local terms in the counterterms $\Delta S^{(1)}$ which are total derivatives or, equivalently, from the finite local anomalous terms of $\bar{\Gamma}^{(1)}$. Qualitatively the reason for this is as follows. The splitting of the quantum effective action into divergent and finite parts is ambiguous and the actual expression for the conformal anomaly becomes dependent on this splitting in the local terms which break local conformal symmetry. In principle, the ambiguity has to be fixed by the choice of a suitable renormalization scheme [5, 7]. However, as we shall see below, in the presence of background scalar this may be a difficult task to accomplish.

The ambiguity in the splitting can be easily understood within the dimensional regularization scheme. The conditions for constructing the counterterm of the $R \phi^{2}$ type are locality and cancellation of the divergent part of the effective action. However, both requirements allow, instead of the minimal choice $h_{1}=\frac{1}{2}\left[\xi-\frac{n-2}{4(n-1)}\right]$ in the $R \phi^{2}$ term of (9), a more general choice

$$
h_{1}=\frac{1}{2}\left[\xi-\frac{n-2}{4(n-1)}+\kappa(n-4)\right],
$$

where $\kappa$ is an arbitrary dimensionless parameter. For any value of $\kappa$ the counterterm is local and it cancels the UV divergence. Hence we meet an ambiguity. The corresponding term in the anomaly (10) depends on $\kappa$

$$
6 h_{1} \square \phi^{2} \rightarrow 6\left[h_{1}+\kappa\right] \square \phi^{2} .
$$

The arbitrariness here is quite similar to the one discussed in [7] for the $\square R$ term. In the last case the anomaly corresponds to the local $\int d^{4} x \sqrt{g} R^{2}$ term in the effective action, and therefore the arbitrariness in the anomaly corresponds to the freedom of adding a local finite $\int d^{4} x \sqrt{g} R^{2}$ term to the classical vacuum action. In 
the present case the anomalous contribution corresponds to the finite term $\int d^{4} x \sqrt{g} R \phi^{2}$. However, there is a very important difference. Adding a finite $\int d^{4} x \sqrt{g} R^{2}$ term to the classical vacuum action does not break any symmetry of the theory in the sector of scalar quantum fields, while adding $\int d^{4} x \sqrt{g} R \phi^{2}$ may break the conformal symmetry of the quantum sector of the theory. The situation with such term is indeed closer to the one with the $\int d^{4} x \sqrt{g} R^{2}$ term in conformal quantum gravity [23] rather than in the semiclassical theory.

From the previous discussions it is also straightforward to understand what is the general structure of the ambiguous terms in any dimension: $\square \phi^{2}$ and $R$ in two dimensions and furthermore

$$
\square \phi^{2}, \quad \square\left(R_{\mu \nu \alpha \beta}^{2}\right)^{\frac{n}{2}-1}, \quad \square\left(R_{\alpha \beta}^{2}\right)^{\frac{n}{2}-1}, \quad \cdots \square R^{\frac{n}{2}-1}
$$

for any even dimension $n$. The corresponding local terms of the finite part of the effective actions may have the form

$$
R \phi^{2}, \quad R\left(R_{\mu \nu \alpha \beta}^{2}\right)^{\frac{n}{2}-1}, \quad R\left(R_{\alpha \beta}^{2}\right)^{\frac{n}{2}-1}, \cdots R^{\frac{n}{2}} .
$$

In the case of background gauge fields there are also extra terms like the Chern-Simons term in 3-dimensions 21, 24.

Let us discuss, for completeness, another possible implementation of local conformal symmetry in dimensional regularization which does not lead to the same result. Following the method introduced in [6] (see also [8, 25]) one could use a different transformation law for the background scalar field (7) and the corresponding value of $\xi(n)$. The peculiarity of this prescription is that $S_{\text {scal }}$ becomes invariant under local conformal transformations in arbitrary dimension and, of course, the same is true for the corresponding counterterms. Thus, although the pure gravitational part of the anomaly is obtained as in the minimal prescription [7], there is no $\phi$ dependent part of the anomaly. The final expression for the conformal anomaly is reduced to

$$
\begin{aligned}
<\mathcal{T}^{\prime}> & =-\left.\frac{1}{\sqrt{\bar{g}}} \frac{\delta \Delta S^{(1)}\left(n ; g_{\mu \nu} e^{2 \sigma}, \phi e^{\frac{2-n}{2} \sigma}\right)}{\delta \sigma}\right|_{\substack{\sigma \rightarrow 0 \\
n \rightarrow 4}} \\
& =\beta_{1} C^{2}+\beta_{2} R^{2}+\beta_{3} E+\beta_{4} \square R,
\end{aligned}
$$

that essentially differs from the correct one (10). There are missing terms which depend on the scalar background field $\phi$. A more exotic renormalization scheme, leading to the total uncontrollable ambiguity in the conformal anomaly, is demonstrated in the Appendix.

\section{SELF-INTERACTING SCALAR FIELD}

The derivation of the effective action for selfinteracting scalar field theory (1) has already been carried out in dimensional regularization [15] and we shall merely analyze the limit relevant for conformal anomaly.
For the sake of simplicity we consider the special case with vanishing values of the coefficients $\tau, \kappa, \rho$ of the odd-power terms in the action (11).

The one-loop effective action is defined, within the background field method, as

$$
\bar{\Gamma}^{(1)}=-\frac{1}{2} \operatorname{Tr} \ln \left[-\hat{1} \square-\hat{1} m^{2}-\hat{P}+\frac{\hat{1}}{6} R\right],
$$

where the hats indicate operators acting in the space of the quantized fields. For the case of the real scalar field $\phi, \hat{1}$ is the image of the delta function and $\hat{P}=-(\xi-$ $1 / 6) R+\lambda \phi^{2} / 2$. The divergent part of effective action can be easily calculated using the standard SchwingerDeWitt technique

$$
\begin{gathered}
\bar{\Gamma}_{\text {scalar }}^{(1) \text { div }}=-\frac{1}{(4 \pi)^{2}(n-4)} \int d^{4} x \sqrt{g}\left\{\frac{\lambda}{12} \square \phi^{2}\right. \\
-\frac{\lambda}{2}\left(\xi-\frac{1}{6}\right) \square R+\frac{1}{180} \square R+\frac{1}{120} C^{2}-\frac{1}{360} E \\
\left.-\frac{1}{2}\left(\xi-\frac{1}{6}\right)^{2} R^{2}+\frac{\lambda}{2}\left(\xi-\frac{1}{6}\right) R \phi^{2}+\frac{\lambda^{2}}{8} \phi^{4}\right\} .
\end{gathered}
$$

Let us notice that all the integrals in this section are four-dimensional, because we use dimensional regularization in the minimal way discussed in the previous section. Alternatively one can apply the proper-time cut-off regularization [7, 12] here, the result is of course the same. Later on we shall see that if instead of dimensional regularization we use the covariant Pauli-Villars regularization [7] the logarithmic divergent part of the action will agree with (14).

The calculation of both divergent and finite part of the effective action has been performed up to the second order in the curvatures $\left(R_{\mu \nu \alpha \beta}, \hat{P}\right)$ using Feynman diagrams on flat backgrounds and also the heat kernel solution [12, 13]. The result is [14, 15]

$$
\begin{aligned}
\bar{\Gamma}_{\text {scalar }}^{(1)} & =\frac{1}{2(4 \pi)^{2}} \int d^{4} x \sqrt{g}\left\{\frac{m^{4}}{2} \cdot\left(\frac{1}{\epsilon}+\frac{3}{2}\right)\right. \\
& +\left(\xi-\frac{1}{6}\right) m^{2} R\left(\frac{1}{\epsilon}+1\right) \\
& +\frac{1}{2} C_{\mu \nu \alpha \beta}\left[\frac{1}{60 \epsilon}+k_{W}(a)\right] C^{\mu \nu \alpha \beta} \\
& +R\left[\frac{1}{2 \epsilon}\left(\xi-\frac{1}{6}\right)^{2}+k_{R}(a)\right] R \\
& -\frac{\lambda}{2 \epsilon} m^{2} \phi^{2}+\phi^{2}\left[\frac{\lambda^{2}}{8 \epsilon}+k_{\lambda}(a)\right] \phi^{2} \\
& \left.+\phi^{2}\left[-\frac{\lambda}{2 \epsilon}\left(\xi-\frac{1}{6}\right)+k_{\xi}(a)\right] R\right\}
\end{aligned}
$$

where

$$
\frac{1}{\epsilon}=\frac{2}{4-n}+\ln \left(\frac{4 \pi \mu^{2}}{m^{2}}\right)-\gamma,
$$

$\gamma$ being the Euler number. The relevant form factors are 
[14, 15]

$$
\begin{aligned}
k_{\lambda}(a) & =\frac{A \lambda^{2}}{4} \\
k_{\xi}(a) & =\lambda\left[\frac{A\left(a^{2}-4\right)}{12 a^{2}}-\frac{1}{36}-A\left(\xi-\frac{1}{6}\right)\right] \\
k_{W}(a) & =\frac{8 A}{15 a^{4}}+\frac{2}{45 a^{2}}+\frac{1}{150}, \\
k_{R}(a) & =A\left(\xi-\frac{1}{6}\right)^{2}-\frac{A}{6}\left(\xi-\frac{1}{6}\right)+\frac{2 A}{3 a^{2}}\left(\xi-\frac{1}{6}\right) \\
& -\frac{A}{18 a^{2}}+\frac{A}{9 a^{4}}+\frac{A}{144}+\frac{1}{108 a^{2}}-\frac{7}{2160} \\
& +\frac{1}{18}\left(\xi-\frac{1}{6}\right),
\end{aligned}
$$

where we used the notations [14]

$$
A=1-\frac{1}{a} \ln \frac{1+a / 2}{1-a / 2}, \quad a^{2}=\frac{4 \square}{\square-4 m^{2}} .
$$

An important difference between the divergences (14) and the divergent part of (15) is that the first expression does include surface terms while the second one does not. Indeed, one can always restore these terms using anomaly.

The conformal limit corresponds to $m^{2} \rightarrow 0$ and $\xi \rightarrow$ $1 / 6$. The useful relations corresponding to the massless limit $m^{2} \rightarrow 0$ are as follows:

$$
a \rightarrow 2, \quad A \sim \frac{1}{2} \log |2-a| \rightarrow \infty, \quad(a-2) A \rightarrow 0 .
$$

Applying the limit (19) to the expression (15) reduces the non-conformal term to

$$
\begin{aligned}
\bar{\Gamma}_{r e g}^{(1)} & =-\frac{1}{2(4 \pi)^{2}} \int d^{4} x \sqrt{g}\left\{\frac{\lambda^{2}}{8} \phi^{2} \log \left(\frac{\square}{4 \pi \mu^{2}}\right) \phi^{2}\right. \\
& +\frac{1}{120} C_{\mu \nu \alpha \beta} \log \left(\frac{\square}{4 \pi \mu^{2}}\right) C^{\mu \nu \alpha \beta} \\
& \left.+\frac{\lambda}{36} \phi^{2} R+\frac{1}{1080} R^{2}\right\} .
\end{aligned}
$$

It is easy to see (using the formulas of previous section) that this result perfectly fits with the divergence (14). The coincidence holds for both metric and metric-scalar local terms. Hence, at this level, the heat-kernel solution does not show any sign of ambiguity discussed in section 2 .

Let us now analyze the explicit emergence of these ambiguities in the framework of Pauli-Villars regularization. In this regularization method the classical action (1) is supplemented with extra Pauli-Villars fields whose interactions with the physical field $\phi$ are given by

$$
\begin{aligned}
S_{\mathrm{reg}} & =\sum_{i=1}^{N} \int d^{4} x \sqrt{-g}\left\{\frac{1}{2} g^{\mu \nu} \partial_{\mu} \varphi_{i} \partial_{\nu} \varphi_{i}+\frac{\xi_{i}}{2} R \varphi_{i}^{2}\right. \\
& \left.-\frac{m_{i}^{2}}{2} \varphi_{i}^{2}-\frac{\lambda}{2} \phi^{2} \varphi_{i}^{2}\right\} .
\end{aligned}
$$

The physical scalar field $\phi$ (also labeled below by $\varphi_{0}$ ) is conformally coupled $(\xi=1 / 6)$ and has bosonic statistics $\left(s_{0}=1\right)$. The $N$ Pauli-Villars fields $\varphi_{i} \quad(i=1, \ldots, N)$ are massive $m_{i}=\mu_{i} M \neq 0$ and may have degeneracy $s_{i}$. These fields can have either bosonic or fermionic statistics. In the first case the degeneracy of the field $s_{i}$ has to by multiplied by the factor of 1 and in the former case by the factor of -2 . We also assume, for the sake of completeness, that the Pauli-Villars regulators might have non-conformal couplings $\xi_{i} \neq 1 / 6$. The regularized effective action of the massless scalar field with conformal coupling $\xi=1 / 6$ is given by

$$
\bar{\Gamma}_{\text {reg }}^{(1)}=\lim _{\Lambda \rightarrow \infty} \sum_{i=0}^{N} s_{i} \bar{\Gamma}_{\mathrm{i}}^{(1)}\left(m_{i}, \xi_{i}, \Lambda\right)
$$

where $\Lambda$ is an auxiliary momentum cut-off.

According to the general prescription, all divergences in the ultraviolet cut-off $\Lambda$ are cancelled out due to the Pauli-Villars conditions

$$
\begin{array}{r}
\sum_{i=1}^{N} s_{i}=-s_{0}=-1 ; \\
\sum_{i=1}^{N} s_{i} \mu_{i}^{2}=0 ; \sum_{i=1}^{N} s_{i}\left(\xi_{i}-\frac{1}{6}\right)=0 ; \\
\sum_{i=1}^{N} s_{i} \mu_{i}^{4}=0 ; \sum_{i=1}^{N} s_{i}\left(\xi_{i}-\frac{1}{6}\right)^{2}=0 .
\end{array}
$$

which are identical to those of the free scalar fields [7]. The first equation (23) cancels out quartic divergences $\Lambda^{4}$, the second and third equations (24) cancel quadratic ones $\Lambda^{2}$ and the last two equations (25) are required to cancel logarithmic divergences $\log \left(\Lambda^{2} / m^{2}\right)$. A simple solution of these equations matching all these requirements is

$$
\begin{gathered}
s_{1}=1, \quad s_{2}=4, \quad s_{3}=-s_{4}=s_{5}=-2 \\
\mu_{1}^{2}=4, \quad \mu_{2}^{2}=3, \quad \mu_{3}^{2}=1, \quad \mu_{4}^{2}=3, \quad \mu_{5}^{2}=4 \\
\text { and } \quad \xi_{i}=\mu_{i}^{2}+\frac{1}{6} .
\end{gathered}
$$

Another possible solution emerges if one takes all $s_{i}, \mu_{i}$ as in (26) and $\xi_{i} \equiv 1 / 6$. At that point we detect an ambiguity in the solution for the regularized effective action.

A compact expression for the effective action is obtained in the limit $M \rightarrow \infty$. In this limit the form factors $k_{W}(a)$ and $k_{R}(a)$ for the auxiliary regulator fields $\varphi_{1, \ldots, 5}$ vanish and the asymptotic form of the remaining 
expression has the form

$$
\begin{aligned}
\bar{\Gamma}_{r e g}^{(1)} & =\frac{1}{2(4 \pi)^{2}} \int d^{4} x \sqrt{g}\left\{\frac{M^{4} \alpha}{2}+M^{2} R \beta\right. \\
& +\left(\delta-\frac{1}{1080}\right) R^{2}-\lambda\left(\frac{1}{36}+\sigma\right) \phi^{2} R \\
& -\frac{\lambda^{2}}{8} \phi^{2} \log \left(\frac{\square}{4 \mathrm{e}^{\eta^{\prime}} M^{2}}\right) \phi^{2} \\
& \left.-\frac{1}{120} C_{\mu \nu \alpha \beta} \log \left(\frac{\square}{4 \mathrm{e}^{\eta} M^{2}}\right) C^{\mu \nu \alpha \beta}\right\},
\end{aligned}
$$

where

$$
\begin{array}{r}
\alpha=\sum_{i=1}^{N} s_{i} \mu_{i}^{4} \ln \mu_{i}^{2}, \\
\beta=\sum_{i=1}^{N} s_{i} \mu_{i}^{2}\left(\xi_{i}-\frac{1}{6}\right) \ln \mu_{i}^{2}, \\
\eta=\frac{2}{3}-\sum_{i=1}^{N} s_{i} \ln \mu_{i}^{2}=\frac{2}{3}+\eta^{\prime}, \\
\sigma=\frac{1}{2} \sum_{i=1}^{N} s_{i}\left(\xi_{i}-\frac{1}{6}\right) \ln \mu_{i}^{2}, \\
\delta=\sum_{i=1}^{N} s_{i}\left(\xi_{i}-\frac{1}{6}\right)^{2} \ln \mu_{i}^{2},
\end{array}
$$

The finite part of the effective action (15) which breaks conformal invariance has two kind of terms. First, the terms which are non-local because of the presence of logarithmic in $\square$ insertions and second those which are local. The last terms essentially reduce to two types, $R \phi^{2}$ and $R^{2}$. The first type of terms have universal coefficients because of their non-locality. The second type of terms depend on arbitrary parameters of the PauliVillars regularization $\delta$ and $\sigma$. These properties are inherited by their descendents in the expression for conformal anomaly in the covariant Pauli-Villars regularization

$$
\begin{aligned}
<\mathcal{T}> & =\frac{1}{(4 \pi)^{2}}\left[\frac{1}{120} C^{2}-\frac{1}{360} E+\frac{\lambda^{2}}{8} \phi^{4}\right. \\
& \left.+\left(\frac{1}{180}-6 \delta\right) \square R+\left(\frac{1}{12}+3 \sigma\right) \square \phi^{2}\right] .
\end{aligned}
$$

The last expression shows that, whereas the coefficient of the $\lambda \phi^{4}$, Weyl and Euler terms in the anomaly are universal, those of $\square R$ and $\square \phi^{2}$ terms are in fact arbitrary, for they depend on the regularization procedure. Qualitatively this is the same ambiguity we have detected in the dimensional regularization case.

The analysis performed above can be easily expanded and we can establish a general structure of the ambiguities. The ambiguous terms of the effective action are always (up to the conformal terms) of the form $R \mathcal{A}(R, \phi)$, where $\mathcal{A}$ is a dimension $n-2$ local operator which induce the anomaly terms of the type $\square \mathcal{A}(R, \phi)$. Indeed, in dimension $n=4$, we have $\mathcal{A}=R$ or $\mathcal{A}=\phi^{2}$, in $n=2$, $\mathcal{A}=$ cte or $\mathcal{A}=\phi^{k}$, however from the above consideration it is clear that in arbitrary dimension $n$ the operator $\mathcal{A}=\phi^{2}$ is always ambiguous.

\section{THE YUKAWA MODEL}

The Yukawa model in curved Euclidean space

$$
S_{Y u k a w a}=i \int d^{4} x \sqrt{g} \bar{\psi}\left(\gamma^{\mu} \nabla_{\mu}-i m-i h \phi\right) \psi .
$$

describes the interaction of fermionic field $\psi$ with a scalar background field $\phi$. Let us denote

$$
\varphi=m+h \phi, \quad \hat{H}=\gamma^{\mu} \nabla_{\mu}-i \varphi .
$$

The one-loop effective action can be calculated in two different ways. The first possibility is to consider

$$
\begin{aligned}
\bar{\Gamma}_{\text {fermion }}^{(1)}\left[g_{\mu \nu}, \phi\right] & =\operatorname{Tr} \ln \left(\hat{H} \cdot \hat{H}_{1}^{*}\right)-\frac{1}{2} \operatorname{Tr} \ln \left(\hat{H}_{1}^{*} \cdot \hat{H}_{1}^{*}\right), \\
\hat{H}_{1}^{*} & =\gamma^{\nu} \nabla_{\nu}
\end{aligned}
$$

and the second corresponds to

$$
\begin{aligned}
\bar{\Gamma}_{\text {fermion }}^{(1)}\left[g_{\mu \nu}, \phi\right] & =\frac{1}{2} \operatorname{Tr} \ln \left(\hat{H} \cdot \hat{H}_{2}^{*}\right) \\
\hat{H}_{2}^{*} & =\gamma^{\nu} \nabla_{\nu}+i \varphi,
\end{aligned}
$$

where we use the known fact $\bar{\Gamma}_{\text {fermion }}^{(1)}$ is an even functional in $\varphi$. Let us notice that the derivation of the second term in (32) can be performed in a standard way (see, e.g. the second reference in [14]).

In the first case (32) the relevant operator is

$$
\begin{aligned}
\hat{H} \cdot \hat{H}_{1}^{*} & =\hat{1} \square+2 \hat{h}^{\alpha} \nabla_{\alpha}+\hat{\Pi}, \\
\hat{h}^{\alpha} & =-\frac{i \varphi}{2} \gamma^{\alpha} \quad \text { and } \hat{\Pi}=-\frac{\hat{1}}{4} R .
\end{aligned}
$$

The calculation of divergences can be performed using standard prescription [26, 27] (see also [4]) and the result is

$$
\begin{aligned}
\bar{\Gamma}_{\text {fermion }}^{(1) \text { div }}\left(\hat{H} \cdot \hat{H}_{1}^{*}\right) & =-\frac{1}{(4 \pi)^{2}(n-4)} \int d^{4} x \sqrt{g}\left\{\frac{k}{2} \square \varphi^{2}\right. \\
& +\frac{1}{30} \square R+2 g^{\mu \nu} \partial_{\mu} \varphi \partial_{\nu} \varphi-2 \varphi^{4} \\
& \left.+\frac{1}{3} R \varphi^{2}+\frac{1}{20} C^{2}-\frac{11}{360} E\right\} \cdot(35)
\end{aligned}
$$

where $k=k_{1}=-8 / 3$.

In the second approach (33) the relevant operator is

$$
\begin{aligned}
\hat{H} \cdot \hat{H}_{2}^{*} & =\hat{1} \square+\hat{\Pi}_{1}, \quad \text { where } \\
\hat{\Pi}_{1} & =\varphi^{2}+i \gamma^{\mu} \varphi_{, \mu}-\frac{\hat{1}}{4} R .
\end{aligned}
$$

The divergences have the same form except that the coefficient $k$ has a different value $k=k_{2}=-4 / 3$. The 
difference in the coefficient $k$ is quite remarkable, for it might indicate to the arbitrariness in the anomalous $R \phi^{2}$ term in the finite part of the effective action. Later on we shall confirm that this is exactly the case.

The operator $\left(\hat{H} \cdot \hat{H}_{1}^{*}\right)$ in the prescription (32) has linear in derivative term and does not admit direct application of the heat-kernel solution [12]. The derivation of the full (with finite part) effective action using the method of [12, 14] gives, in the case of the prescription (33), the following result:

$$
\begin{aligned}
\bar{\Gamma}_{\text {fermion }}^{(1)}\left(\hat{H} \cdot \hat{H}_{2}^{*}\right) & =\frac{1}{(4 \pi)^{2}} \int d^{4} x \sqrt{g}\left\{-m^{4}\left(\frac{1}{\epsilon}+\frac{3}{2}\right)\right. \\
& +\frac{1}{6} m^{2} R\left(\frac{1}{\epsilon}+1\right)+\frac{1}{2} R k_{R}^{f}(a) R \\
& -2 m^{2}\left(\varphi^{2}-m^{2}\right)\left(\frac{1}{\epsilon}+1\right) \\
& +\frac{1}{4} C_{\mu \nu \alpha \beta}\left[\frac{1}{10 \epsilon}+k_{W}^{f}(a)\right] C^{\mu \nu \alpha \beta} \\
& +\frac{1}{2}\left(\nabla_{\alpha} \varphi\right)\left[\frac{2}{\epsilon}+4 A\right]\left(\nabla^{\alpha} \varphi\right) \\
& +\frac{1}{2}\left(\varphi^{2}-m^{2}\right)\left[\frac{1}{3 \epsilon}+\frac{2}{9}+\frac{8 A}{3 a^{2}}\right] R \\
& \left.-\frac{1}{2}\left(\varphi^{2}-m^{2}\right)\left[\frac{2}{\epsilon}+4 A\right]\left(\varphi^{2}-m^{2}\right)\right\} .
\end{aligned}
$$

The higher derivative form factors $k_{W}^{f}$ and $k_{R}^{f}$ can be found in the second reference in [14]. The change $\varphi^{2} \rightarrow$ $\left(\varphi^{2}-m^{2}\right)=h^{2} \phi^{2}+2 m h \phi$ is due to the fact we have to settle $m^{2}$ into exponential of the heat kernel representation for the effective action [14].

The massless limit (19) of the finite part of the one loop effective action (37) leads to the following expression for the non-conformal term:

$$
\begin{aligned}
\bar{\Gamma}_{r e g}^{(1)} & =-\frac{1}{2(4 \pi)^{2}} \int d^{4} x \sqrt{g}\left\{\frac{1}{90} R^{2}-\frac{2 h^{2}}{9} \phi^{2} R\right. \\
& -2 h^{4} \phi^{2} \log \left(\frac{\square}{4 \pi \mu^{2}}\right) \phi^{2} \\
& +\frac{1}{20} C_{\mu \nu \alpha \beta} \log \left(\frac{\square}{4 \pi \mu^{2}}\right) C^{\mu \nu \alpha \beta} \\
& +2 h^{2}\left(\nabla_{\alpha} \phi\right) \log \left(\frac{\square}{4 \pi \mu^{2}}\right)\left(\nabla^{\alpha} \phi\right) \\
& \left.+\frac{h^{2}}{3} \phi^{2} \log \left(\frac{\square}{4 \pi \mu^{2}}\right) R\right\} .
\end{aligned}
$$

which is quite similar to the result for the scalar case (20). There are non-local finite terms which are in one to one correspondence with the local UV divergent terms. These terms were cancelled by counterterms in a momentumsubtraction renormalization scheme (see [14] for a detailed discussion). On the top of that, we have two usual local terms $\int \sqrt{g} R^{2}$ and $\int \sqrt{g} R \phi^{2}$. At this level no ambiguity is observed.

More complete analysis can be performed in the framework of Pauli-Villars regularization, in a way similar to that described in the previous section. Our purpose is to detect the ambiguity in the local terms $\int \sqrt{g} R^{2}$ and $\int \sqrt{g} R \phi^{2}$, these ambiguities are expected to be similar to the ones we met for the scalar case. In order to use this analogy we need to introduce some parameters similar to the non-minimal ones $\xi_{i}$.

In order to get the most general ambiguity we shall introduce PV regulator fields $\psi_{i}$ with bilinear couplings like in (36) to include non-minimal couplings

$$
\begin{aligned}
\left(\hat{H} \cdot \hat{H}_{2}^{*}\right)_{(i)} & =\hat{1} \square+\hat{\Pi}_{1 i}, \quad \text { where } \\
\hat{\Pi}_{1 i} & =\varphi_{i}^{2}+i \gamma^{\mu} \varphi_{i, \mu}+\left(\chi_{i}-\frac{1}{4}\right) \hat{1} R
\end{aligned}
$$

where $\varphi_{i}=h \phi+M_{i}, M_{i}=\mu_{i} M$ being the mass of the regulator fields, $M$ is the regularization scale parameter and $\mu_{i}$ dimensionless parameters which can be defined from the condition of cancelling the divergences. Finally, $\chi_{i}$ are new non-minimal parameters, which may be safely introduced for the massive regulators. The operators (39) act in the space of Dirac fermions, but may have either fermionic or bosonic statistics. The corresponding multiplicities are $d_{i}=(1,-2)$, like in the scalar case.

$$
\begin{aligned}
\bar{\Gamma}_{r e g} & =-\frac{1}{2} \operatorname{Tr} \log \left(\hat{H} \cdot \hat{H}_{2}^{*}\right) \\
& +\frac{1}{2} \sum_{i=1}^{N}(-1)^{d_{i}} \operatorname{Tr} \log \left(\hat{H} \cdot \hat{H}_{2}^{*}\right)_{(i)} .
\end{aligned}
$$

The Pauli-Villars conditions for $s_{i}$ and $\mu_{i}$ are similar to (23)-(25), the only difference is the sign of the contribution of the massless physical field, which is opposite compared to the scalar case

$$
\begin{aligned}
\sum_{i=1}^{N} s_{i} & =-s_{0}=+1 \\
\sum_{i=1}^{N} s_{i} \mu_{i}^{2} & =\sum_{i=1}^{N} s_{i} \chi_{i}=\sum_{i=1}^{N} s_{i} \mu_{i}^{4}=\sum_{i=1}^{N} s_{i} \chi_{i}^{2}=0
\end{aligned}
$$

and also we have here factors $\chi_{i}$ instead of $\xi_{i}-1 / 6$.

The solution of equations (41) is opposite in sign to the one of (26).

$$
\begin{gathered}
s_{1}=-1, \quad s_{2}=-4, \quad s_{3}=-s_{4}=s_{5}=2, \\
\mu_{1}^{2}=4, \quad \mu_{2}^{2}=3, \quad \mu_{3}^{2}=1, \quad \mu_{4}^{2}=3, \quad \mu_{5}^{2}=4 \\
\text { and } \quad \chi_{i}=\mu_{i}^{2} .
\end{gathered}
$$

Exactly as in the scalar case, another solution emerges if taking the same $s_{i}, \mu_{i}^{2}$ and $\chi_{i} \equiv 0$.

Finally, we arrive, in the massless limit $m \rightarrow 0$, at the following expression for the conformal anomaly

$$
\begin{aligned}
<\mathcal{T}> & =\frac{1}{(4 \pi)^{2}}\left[\frac{1}{20} C^{2}-\frac{11}{360} E+\left(\frac{1}{30}-6 \delta\right) \square R\right. \\
& +2 h^{2} \nabla_{\alpha} \phi \nabla^{\alpha} \phi-2 h^{4} \phi^{4}+\frac{h^{2}}{3} R \phi^{2} \\
& \left.-\left(\frac{2}{3}+\sigma\right) h^{2} \square \phi^{2}\right],
\end{aligned}
$$


where $\sigma$ and $\delta$ are parameters depending on details of the Pauli-Villars regulators. This dependence indicates the ambiguities of the coefficients of the total derivative terms $\square \phi^{2}$ and $\square R$. Qualitatively, the ambiguity is the same as in the scalar case.

\section{CONCLUSIONS}

We have investigated a problem of conformal anomaly and the corresponding ambiguity in the theory of quantized matter fields on the background which consists of the space-time metric and an additional scalar field. The anomaly can be evaluated via different methods and perhaps the most simple one is based on the dimensional regularization. However, the renormalization schemes based on this regularization allow a much larger ambiguity in the anomalous part of effective action. A similar ambiguity can be also observed in covariant Pauli-Villars regularizations. Except the specially designed artificial renormalization scheme described in the Appendix, the ambiguity concerns only the local sector of the effective action, while the non-local part of it is well defined. In this respect our results are quite similar to the ones obtained earlier in [7] for the purely metric background case. Although there is a serious difference between the two cases. The ambiguity in the pure metric case concerns only the vacuum sector, that is the effective action of external metric field. In the present case of a metricscalar background the ambiguity concerns also the action of quantized scalar field. Hence the effect of this ambiguity is much stronger. If one attempts to fix the problem by introducing the special renormalization condition, the theory becomes conformally non-invariant already at the classical level. In this respect the theory of conformal scalar field is closer to quantum conformal gravity [23], rather than to the theory of free conformal matter on purely metric background.

Let us notice that the renormalization scheme ambiguities are common in the higher loop corrections to the $\beta$-functions and therefore to the trace anomaly. For example, they have been discussed in [29, 30] for the case of quantum field theory in curved space-time. The fundamental difference between our results and the ones of the mentioned papers is that we have found the origin of the trace anomaly ambiguity which shows up already at the one-loop level. Furthermore, we have clarified the existing mismatch between the anomalous violations of global and local conformal symmetries and found that the ambiguities can arise not only within dimensional regularization, but also for general covariant Pauli-Villars regularizations.

Finally, the main lesson we learned from exploring the ambiguity in the quantum violation of local conformal symmetry is that this symmetry can not be exact even at the classical level. The model with local conformal symmetry does not enable one to obtain a consistent theory at quantum level, because the quantum corrections are plagued by ambiguities. Our consideration also leaves open the possibility to regard the conformal symmetry as an approximation. In this case the conformal anomaly must be seen as a useful way to evaluate the effective action and the corresponding ambiguities may be easily fixed by implementing the corresponding renormalization conditions on the coefficients of local non-conformal terms in the renormalized action of the theory. The approximate character of conformal symmetry will manifest itself in a hierarchy of the parameters of the starting action. The parameters in the non-conformal sector must be some orders of magnitude smaller than the ones in the conformal sector. In this case local non-conformal terms will be indeed modified by the quantum (mainly anomalous) contributions, while the ambiguities can be easily fixed through the renormalization conditions in the non-conformal corner of the theory.

Acknowledgments. The work of the authors has been partially supported by the Spanish CICYT (grant FPA2004-02948) and DGIID-DGA (grant2005-E24/2) (M.A.), by research grants from $\mathrm{CNPq}$ (Brazil) and FAPEMIG (Minas Gerais, Brazil) (G.B.P. and I.Sh.), by the post-doctoral PRODOC fellowship from CAPES (G.B.P.) and by the long-term research fellowships from CNPq and ICTP (I.Sh.). I.Sh. is thankful to the Departamento de Física Teórica at the University of Zaragoza for support and kind hospitality.

\section{APPENDIX A: REMARK ABOUT MORE GENERAL AMBIGUITIES IN DIMENSIONAL REGULARIZATION}

The arbitrariness described in section 2 concerns only the local finite term $\int d^{4} x \sqrt{g} R \phi^{2}$ in the effective action and corresponding $\square \phi^{2}$ term in the anomalous trace $\langle\mathcal{T}\rangle$. On the other hand, the presence of scalar field with a non-trivial transformation law enables one to design renormalizations schemes which make the arbitrariness in the anomaly make the arbitrariness in the anomaly very much larger than that derived via dimensional regularization in a usual way. Let us notice that the conditions for the counterterms formulated in section 2 (locality plus cancellation of the divergent part of the effective action) can be satisfied in a great variety of different ways in the presence of a scalar field. For example, in the scalar field sector one can keep the transformation of scalar field the same (3), independent on the dimension $n$. Then the derivation of anomaly in this sector will be essentially the same as for the gravitational sector and the anomaly $\langle\mathcal{T}\rangle$ will be just proportional to the divergent part of effective action, plus a standard $\square \phi^{2}$-type arbitrariness described above. On the other hand, in the purely gravitational field one can use scalar field to eliminate the anomaly completely. Let us choose 
the counterterm for the Weyl term in the form

$$
\Delta S_{W e y l}=\frac{\beta_{1}}{n-4} \int d^{n} x \sqrt{g} \phi^{n-4} C^{2},
$$

where we took the simplest transformation law (3) of the scalar field for simplicity 34]. It is obvious that this counterterm is conformal invariant and therefore using the standard procedure of deriving anomaly from [6] does not produce anomaly et all. The same is true also for other anomalous terms. Therefore, the combination of the dimensional regularization and scalar field makes the whole anomaly arbitrary, not only the part corresponding to the local sector of the one-loop effective action.

There are several reasons why such an ambiguous renormalization scheme is unphysical. The dependence $\phi^{n-4}$ is non-analytic on the scalar field $\phi$ for non-integer values of $n$ and for such a reason it can never be generated by radiative perturvative corrections. Indeed, in perturbation theory Feynman graphs have a definite integer number of external legs $\left(\phi^{\prime} s\right)$ which can not be a frac- tional number (see, however, Ref. [28] for scenarios with emergence of non-perturbative non-analiticities). On the other hand the form of the counterterms does not need to follow the form of the original quantum corrections, but only satisfy the requirements formulated above. Nevertheless, the scheme based on (A1) is very artificial and that the resulting "total" ambiguity does not correspond to the direct calculations of a finite part of the effective action. In general, the existence of the scheme described above just shows that the dimensional regularization is not an appropriate instrument to derive the conformal anomaly, especially if the scalar field is present.
[1] A. D. Linde, Phys. Lett. 108B (1982) 389; A. Albrecht and P. J. Steinhardt, Phys. Rev. Lett. 48 (1982) 1220.

[2] R.D. Peccei, J. Solà, C. Wetterich, Phys. Lett. B195 (1987) 183; C. Wetterich, Nucl. Phys. B 302 (1988) 668; J. Solà, Phys. Lett. B 228 (1989) 317; ibid., Int. J. Mod. Phys. A5 (1990) 4225.

[3] R.R. Caldwell, R. Dave, P.J. Steinhardt, Phys. Rev. Lett. 80 (1998) 1582; P.J.E. Peebles, B. Ratra, Astrophys. J. Lett. 325 L17 (1988); P.J.E. Peebles, B. Ratra, Rev. Mod. Phys. 75(2003) 599, astro-ph/0207347; A. R. Liddle, Rev. Mod. Phys. 75(2003) 599, astro-ph/0207347; C. Armendariz-Picon, V. Mukhanov, P.J. Steinhardt, Phys. Rev. D 63 (2001) 103510.

[4] I.L. Buchbinder, S.D. Odintsov and I.L. Shapiro, Effective Action in Quantum Gravity (IOP Publishing, Bristol, 1992).

[5] N.D. Birrell and P.C.W. Davies, Quantum Fields in Curved Space (Cambridge Univ. Press, Cambridge, 1982).

[6] M.J. Duff, Nucl. Phys. 125B (1977) 334;

S. Deser, M.J. Duff and C. Isham, Nucl. Phys. 111B (1976) 45.

[7] M. Asorey, E.V. Gorbar and I.L. Shapiro, Class. Quant. Grav. 21 (2003) 163.

[8] L.S. Brown and J.C. Collins, Ann. Phys. 130 (1980) 215.

[9] R.J. Reigert, Phys.Lett. 134B (1980) 56.

[10] E.S. Fradkin and A.A. Tseytlin, Phys.Lett. 134B (1980) 187.

[11] M.J. Duff, Class.Quant.Grav. 11 (1994) 1387.

[12] A.O. Barvinsky and G.A. Vilkovisky, Nucl. Phys. 333B (1990) 471.

[13] I. G. Avramidi, Yad. Fiz. (Sov. Journ. Nucl. Phys.) 49 (1989) 1185.

[14] E.V. Gorbar and I.L. Shapiro, JHEP 02 (2003) 021; 06 (2003) 006; E.V. Gorbar, Nucl. Phys. B Proceedings Supplement, 127 (2004) 162.

[15] G. de Berredo-Peixoto, E.V. Gorbar, I.L. Shapiro, Class.
Quant. Grav., 21 (2004) 2281; hep-th/0311229.

[16] A. Iorio, L. O'Raifeartaigh, I. Sachs and C. Wiesendanger, Nucl. Phys. 495B (1997) 433.

[17] A.O.Barvinsky, Yu.V.Gusev, G.A.Vilkovisky and V.V.Zhytnikov, Nucl. Phys. 439B (1995) 561.

[18] I.L. Buchbinder, Theor. Math. Phys. 61 (1984) 393.

[19] S.W. Hawking, Comm. Math. Phys. 55 (1977) 133.

[20] I.L. Shapiro, J. Solà. Phys. Lett. 530B (2002) 10; A.M. Pelinson, I.L. Shapiro, F.I. Takakura, Nucl. Phys. 648B (2003) 417.

[21] M. Asorey, F. Falceto, J.L. López and G. Luzón, Nucl. Phys. 429B (1994) 344.

[22] J.L. López, Universalidad y Regularización Ultravioleta en Teorías Gauge (Ph.D. dissertation, Zaragoza University, 1995).

[23] G. de Berredo-Peixoto and I.L. Shapiro, Phys. Rev. D70 (2004) 044024; hep-th/0307030.

[24] M. Asorey, F. Falceto, J.L. López and G. Luzón, Phys. Rev. D 49 (1993) 5377

[25] S.J. Hathrell, Ann. Phys. 139 (1982) 136; 142 (1982) 34. , Ann. Phys. 139 (1982) 136; 142 (1982) 34.

[26] t'Hooft G. and Veltman M., Ann. Inst. H. Poincare A20 (1974) 69.

[27] A.O. Barvinsky and G.A. Vilkovisky, Nucl. Phys. 191B (1981) 237.

[28] M. Asorey, D. García-Álvarez, J.L. López, In From Fields to Strings: Circumnavigating Theoretical Physics, Eds, M. Shifman, A. Vainshtein, and J. Wheater, World Sci., Vol. 1, Singapore (2005) 559.

[29] I. Jack, Nucl. Phys. 253 (1985) 323.

[30] H. Osborn, Nucl. Phys. 363 (1991) 486.

[31] We use the Euclidean metric $\eta_{\mu \nu}=\operatorname{diag}(++++)$ and the definition $R_{\mu \nu}=\partial_{\lambda} \Gamma_{\mu \nu}^{\lambda}-\partial_{\nu} \Gamma_{\mu \lambda}^{\lambda}+\Gamma_{\mu \nu}^{\sigma} \Gamma_{\sigma \lambda}^{\lambda}-\Gamma_{\mu \lambda}^{\sigma} \Gamma_{\sigma \nu}^{\lambda}$. We avoid using letter $G$ for the Gauss-Bonnet invariant because it may be confused with the Newton constant.

[32] Later on, in section 4, we shall see that in some situations these terms are indeed necessary. 
[33] The relation between local and global conformal symmetries have been discussed, in particular, in [16].

[34] Choosing another version of the transformation law in $n \neq 4$ one can always adjust the power in (A1) such that the result for the anomaly would be the same. 